\title{
Comparison of gene expression profiles in aortic dissection and normal human aortic tissues
}

\author{
LIANG ZHANG, CUNTAO YU, QIAN CHANG, XINJIN LUO, JUNTAO QIU and SHEN LIU
}

\begin{abstract}
State Key Laboratory of Cardiovascular Disease, Aortic Surgery Center, Fuwai Hospital, National Center for Cardiovascular Disease, Chinese Academy of Medical Science and Peking Union Medical College, Beijing 100037, P.R. China
\end{abstract}

Received April 2, 2016; Accepted July 6, 2016

DOI: $10.3892 /$ br. 2016.740

\begin{abstract}
The aim of the present study was to compare the gene expression profiles in aortic dissection (AD) and healthy human aortic tissue samples by DNA microarray analysis in order to screen the differential genes. In total, five AD and four healthy aortic specimens were selected; the total RNA was extracted and reverse transcribed into cDNA and in vitro transcribed into aRNA, followed by microarray hybridization for analysis. Thereafter, the transcription levels of six differential genes, myosin light chain kinase $(M Y L K)$, polycystin 1 , transient receptor potential channel interacting $(P K D-1)$, myosin heavy chain 11 (MYH11), superoxide dismutase 3, extracellular (SOD3), filamin A (FLNA), and transgelin $(T A G L N)$, screened from the expression profiles were quantitatively verified. Compared with the healthy aortic specimens, a total of 1,661 genes in the AD group demonstrated more than 2-fold differential expression, of which 997 genes were upregulated and 664 genes were downregulated. Thereafter, six AD-associated genes that showed downregulation in the microarray assay were selected for quantitatively verifying the gene transcription level using reverse transcription-quantitative polymerase chain reaction (RT-qPCR), which confirmed their downregulation compared with the healthy aortic tissue genes; of the six genes, the expression levels of MYLK, PKD-1, $M Y H 11$, SOD3 and TAGLN were significantly downregulated $(\mathrm{P}<0.05)$, while the expression of FLNA was not significantly downregulated $(\mathrm{P}>0.05)$. Thus, whole genome microarray may be used to screen differentially expressed genes between AD and healthy aortic tissues. When used in combination with RT-qPCR validation, this method may provide novel strategies for investigating AD.
\end{abstract}

Correspondence to: Dr Cuntao Yu, State Key Laboratory of Cardiovascular Disease, Aortic Surgery Center, Fuwai Hospital, National Center for Cardiovascular Disease, Chinese Academy of Medical Science and Peking Union Medical College, 167 Beilishi Road, Beijing 100037, P.R. China

E-mail: cuntaoyu@126.com

Key words: aortic dissection, gene expression profile, reverse transcription-polymerase chain reaction

\section{Introduction}

Aortic dissection (AD) is the most common life-threatening cardiovascular disorder, with an annual incidence ranging from 5 to 30 per 100,000 individuals (1). This condition has a high mortality (2); 3\% of the patients succumb suddenly at the time of onset, and $50 \%$ within two days, and the 1-month mortality is as high as $90 \%$ in patients who do not undergo surgery (3). With improvements in the overall standard of living causing individuals to live longer, the incidence of AD is increasing, thereby causing a decline in medical resources and social wealth. A detailed investigation on the molecular mechanism underlying AD may facilitate with delaying or even reversing arterial wall reconstruction, consequently reducing the incidence of AD and improving patient prognosis.

The pathogenesis of AD remains unclear; several genes are considered to be involved in its occurrence. For example, patients with Marfan syndrome suffer from deficiency of the glycoprotein, fibrillin-1 (4), while patients with Ehlers-Danlos syndrome have abnormal type-III precollagen (5), such as deletions or bona fide amino acid substitutions, which cause delayed formation and destabilization of the collagen triple helix and, as a consequence, reduced secretion of the molecule, which leads to weakened arterial walls. Such patients may suffer from $\mathrm{AD}$ at a particularly young age; however, the majority of patients with $\mathrm{AD}$ do not exhibit such explicit syndromes. Thus, a detailed investigation of the genes associated with the pathogenesis of AD may help with the early identification and control of this disease by modifying the genes, and via other methods prior to the onset of AD.

In the present study, human whole genome microarray was employed to screen differential genes from AD and normal aortic tissue samples. In addition, reverse transcription-quantitative polymerase chain reaction (RT-qPCR) was adopted to verify the downregulated genes, and the results were compared with the results of microarray analysis in order to investigate the role of differentially expressed genes screened by gene microarray in the pathogenesis of AD.

\section{Materials and methods}

Tissue sampling. Five AD specimens were obtained from patients with non-hereditary AD who were undergoing cardiovascular surgery in Fuwai Hospital (Beijing, China) during 
the time period from July 2014 to December 2014. Patients with familial AD and those who had aortic diseases caused by gene mutation were excluded. Four control specimens were obtained from age-matched patients undergoing heart surgery for non-aortic diseases. The fresh tissues were rinsed with cold phosphate-buffered saline to remove the blood and thrombus on the surface, quickly frozen in liquid nitrogen, and stored at $-80^{\circ} \mathrm{C}$ until use. The present study was approved by the medical ethics committee of Fuwai Hospital and written informed consent was obtained from all patients.

Total RNA extraction and quality test. Total RNA was extracted using TRIzol reagent (Invitrogen; Thermo Fisher Scientific, Inc., Waltham, MA, USA) and purified using the RNeasy mini kit (Qiagen China Co., Ltd., Shanghai, China), according to the manufacturer's instructions. The purity of the total RNA was tested using a NanoDrop 2000 spectrophotometer (Thermo Fisher Scientific, Inc., Wilmington, DE, USA) with $2 \mu 1$ total RNA per sample, and integrity was tested using an Agilent 2100 BioAnalyzer (Agilent Technologies, Inc., Santa Clara, CA, USA).

Microarray hybridization, scanning and analysis. Using RNA as templates, single-strand and double-strand cDNAs were synthesized using the One-cycle cDNA Synthesis kit (Affymetrix, Inc., Santa Clara, CA, USA), followed by combining, washing and elution steps to purify the cDNA. Thereafter, biotin-marked aRNA was in vitro transcribed using an RNA transcription kit (Qiagen China Co., Ltd.) with double-stranded cDNA as templates, followed by fragment processing. Subsequently, the qualified cDNA was pre-hybridized with the GeneChip ${ }^{\circledR}$ Human Genome U133 Plus 2.0 Array (Affymetrix, Inc.) at $45^{\circ} \mathrm{C}$ for $10 \mathrm{~min}$ using a Hybridization Oven 640 (Affymetrix, Inc.). Subsequently, the hybridization solution was removed and an equal volume of prepared hybridization solution was added for hybridization at $45^{\circ} \mathrm{C}$ for $16 \mathrm{~h}$. The microarray was then eluted and stained using Fluidics Station 450, and was ultimately scanned using the GeneChip ${ }^{\circledR}$ Scanner 3000 (Affymetrix, Inc.), after which the scanned images and raw data were processed using the Affymetrix ${ }^{\circledR}$ GeneChip $^{\circledR}$ Command Console $^{\circledR}$ operating system (Affymetrix, Inc.).

Microarray data processing and bioinformatics analysis. Microarray data were analyzed using GCOSvL.4 software according to the standard procedures provided by Affymetrix, the principle and steps of which were referred from the documentation 'Genechip Expression Analysis-Data Analysis Fundamentals'. The microarray data was analyzed using the Gene Ontology (GO; http://www.geneontology. org/gene-associations/) and Kyoto Encyclopedia of Genes and Genomes (KEGG; http://www.kegg.jp/) databases. GO terms were collected from the Gene Ontology and National Center for Biotechnology Information (www.ncbi.nlm. nih.gov/gene/data/GO) databases. Based on this analysis, the five AD specimens and four healthy aortic specimens were orthogonally compared to determine the differentially expressed genes. The ratio of fluorescence signal intensity of AD to normal aortic tissue samples was calculated and transformed to the base 2 logarithm, where a positive value was referred to as an upregulated gene ( $\geq \pm 2$ represented statistically significant upregulation) and a negative value referred to a downregulated gene ( $\leq \pm 2$ represented statistically significant downregulation).

Validation of expression profiles by $R T-q P C R$. Six genes with significant $(\mathrm{P}<0.05)$ differential expressions [myosin light chain kinase $(M Y L K)$, polycystin 1, transient receptor potential channel interacting $(P K D-1)$, myosin heavy chain 11 (MYH11), superoxide dismutase 3, extracellular (SOD3), filamin A (FLNA) and transgelin (TAGLN)] were selected to verify the microarray results using RT-qPCR. The microarray assay and RT-qPCR were performed using the same batch of specimens. The RNAs were extracted using TRIzol reagent, reverse transcribed using Moloney murine leukemia virus (Takara Biotechnology Co., Ltd., Shiga, Japan), and separately subjected to PCR amplification. The primers were synthesized by Invitrogen (Thermo Fisher Scientific, Inc.) using GAPDH as the reference gene. The primer sequences, amplification length and annealing temperature are presented in Table I. The reaction procedure was as follows: Initial denaturation at $94^{\circ} \mathrm{C}$ for $5 \mathrm{~min}$; followed by 35 cycles for denaturation at $94^{\circ} \mathrm{C}$ for $30 \mathrm{sec}$, annealing for $30 \mathrm{sec}$ and elongation at $72^{\circ} \mathrm{C}$ for $30 \mathrm{sec}$; each sample was assayed in triplicate. A temperature range of $65-95^{\circ} \mathrm{C}$ was selected for drawing melting curves.

Statistical analysis. Data were analyzed using SPSS 21.0 software (IBM SPSS, Armonk, NY, USA). All measurement data are reported as the mean \pm standard deviation. Comparisons involving measurement data and rates were performed between groups using paired Student's t-tests and $\chi^{2}$ analysis, respectively. $\mathrm{P}<0.05$ was considered to indicate a statistically significant difference. As per microarray analyses, the target gene expression was considered to increase when the ratio of its expression in the AD tissue/healthy aorta tissue was $\geq 2$-fold, and was considered to decrease when the ratio was $\leq 0.5$.

\section{Results}

Quality testing of total RNA. The A260/A280 value was found to range from 1.8 to 2.2, the optical density (OD)260/OD230 value was found to range from 1.5 to 2.0 , and the RNA integrity index was $>6.0$. In addition, the results demonstrated absence of genomic DNA contamination and good RNA integrity; furthermore, the total RNA $28 \mathrm{~s} / 18 \mathrm{~s}$ peak area ratio of the nine specimens was $\sim 2: 1$, indicating that the RNA accorded with the requirement for microarray hybridization.

Screening of the differentially expressed genes by gene expression microarray. Microarray hybridization revealed that, compared with normal aortic tissues, a total of 1,661 genes in the dissected aortic tissues exhibited greater than 2-fold differential expression (Fig. 1), of which 997 genes were upregulated and 664 genes were downregulated.

GO analyses of differential genes. The screened genes with greater than 2-fold differential expression were subjected to GO analyses. These genes were divided into three categories: Biological process, cellular component and molecular 
Table I. Primer sequences.

\begin{tabular}{lllcc}
\hline $\begin{array}{l}\text { Target } \\
\text { gene }\end{array}$ & \multicolumn{1}{c}{$\begin{array}{c}\text { Upstream } \\
\text { primer }\left(5^{\prime}-3^{\prime}\right)\end{array}$} & \multicolumn{1}{c}{$\begin{array}{c}\text { Downstream } \\
\text { primer }\left(5^{\prime}-3^{\prime}\right)\end{array}$} & $\begin{array}{c}\text { Length } \\
\text { of product }(\mathrm{bp})\end{array}$ & $\begin{array}{c}\text { Annealing } \\
\text { temperature }\left({ }^{\circ} \mathrm{C}\right)\end{array}$ \\
\hline PKD-1 & GGACCATCAACGACAAGCAG & CCATCCCCAAAGTTCCACAG & 280 & 58 \\
MYH11 & TGTGTCGTGGCTACTTG & ATTCTCTGCCTTCTGCT & 233 & 52 \\
MYLK & TCTCATTTACCATTCTGATGGC & GCGCATTCAAAGCTTTTTTC & 169 & 55 \\
SOD3 & TACCGAAACACCCCGCTCA & TGCCAAACATTCCCCCAAA & 325 & 55 \\
FLNA & ACGTGCTTCACCAGGATCTC & CCTGGCAGCTACCTCATCTC & 353 & 58 \\
TAGLN & ATGGCCAACAAGGGTCCATCC & TCCATCTGCTTGAAGACCATG & 275 & 53 \\
GAPDH & ATGGCCAACAAGGGTCCATCC & TCCATCTGCTTGAAGACCATG & 275 & 55 \\
\hline
\end{tabular}

PKD-1, polycystin 1, transient receptor potential channel interacting; MYH11, myosin heavy chain 11; MYLK, myosin light chain kinase; SOD3, superoxide dismutase 3, extracellular; FLNA, filamin A; TAGLN, transgelin; GAPDH, glyceraldehyde-3-phosphate dehydrogenase.

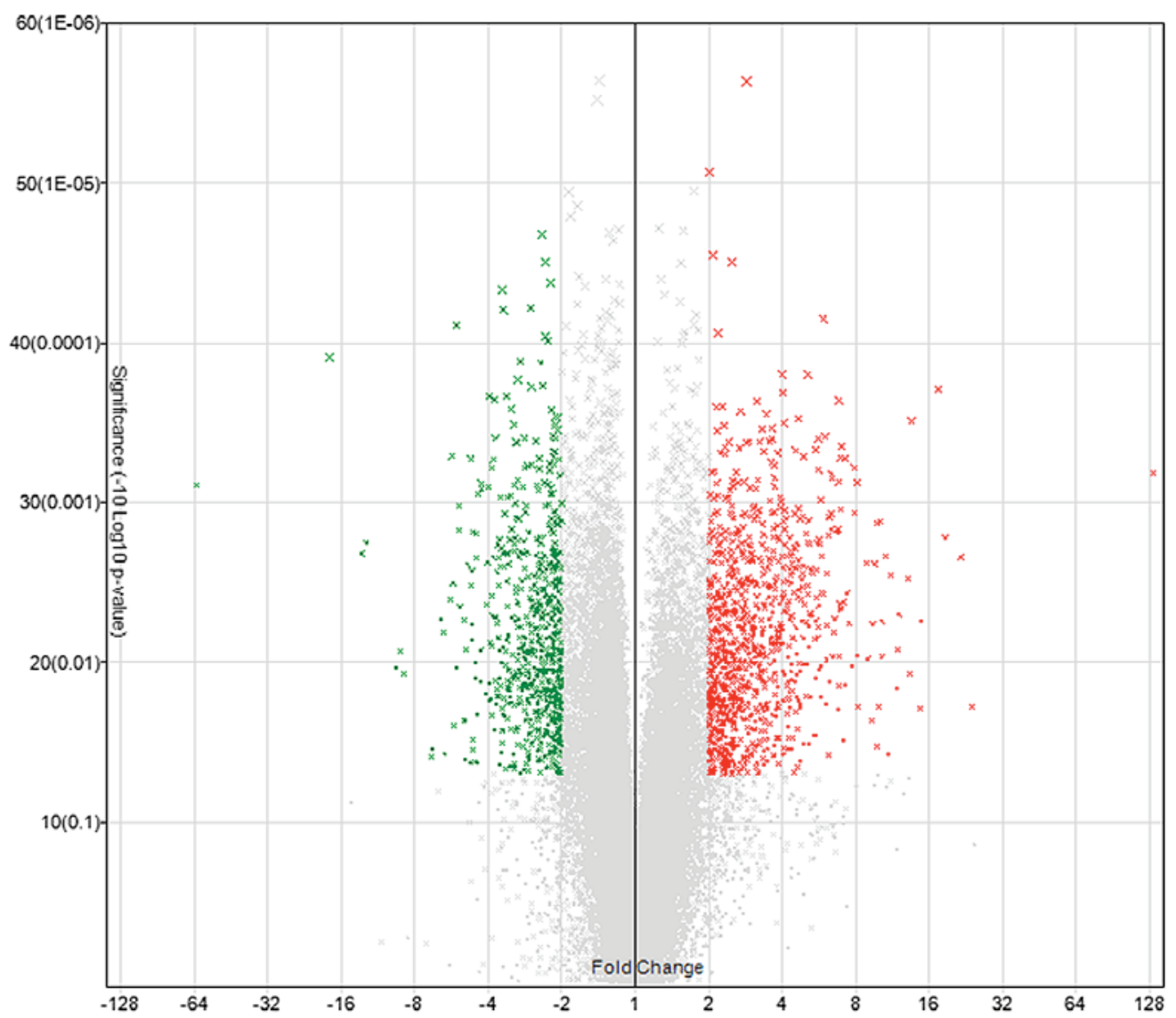

Figure 1. Volcano plot for differentially expressed genes between the aortic dissection and normal aortic tissue samples.

function. The differential genes predominantly involved in cellular immune response, inflammatory reaction, intracellular signaling cascades, and regulation of cell proliferation, cell death and apoptosis, and cell cycle were classified in the category of biological process. The genes that showed significant expression in the cytoplasmic vesicle, myosin complex and extracellular matrix were assigned to the cellular component category. The genes that were primarily involved in actin binding, cytoskeletal protein binding, enzyme binding, calmodulin binding, nucleoside binding, ATP binding, and activation of protein dimer, GTPase, serine/serine protein kinase, and peptase were assigned to the molecular function category (Table II).

Biological pathways for the differentially expressed genes. The central tendencies of the differentially expressed genes in the pathways were determined by the P-value, where a smaller P-value referred to a stronger central tendency. The following signaling pathways were found to be associated with the most differentially expressed genes within the threshold range: Fc $\gamma$ R-mediated phagocytosis, chemokine signaling pathway, complement and coagulation cascade, 
Table II. GO functional analysis of differentially expressed genes.

\begin{tabular}{|c|c|c|c|}
\hline GO ID & GO term & Genes associated with the $\mathrm{GO}$ term, $\mathrm{n}$ & P-value \\
\hline GO:0006955 & Immune response & 125 & $5.67 \mathrm{E}-28$ \\
\hline GO:0009611 & Response to wounding & 93 & $8.62 \mathrm{E}-20$ \\
\hline GO:0006954 & Inflammatory response & 67 & $5.28 \mathrm{E}-18$ \\
\hline GO:0006952 & Defense response & 97 & $2.14 \mathrm{E}-17$ \\
\hline GO:0048584 & Positive regulation of response to stimulus & 44 & $1.84 \mathrm{E}-10$ \\
\hline GO:0050778 & Positive regulation of immune response & 33 & $2.75 \mathrm{E}-10$ \\
\hline GO:0007242 & Intracellular signaling cascade & 135 & $2.79 \mathrm{E}-10$ \\
\hline GO:0045321 & Leukocyte activation & 41 & $1.47 \mathrm{E}-08$ \\
\hline GO:0007155 & Cell adhesion & 82 & 4.57E-08 \\
\hline GO:0042127 & Regulation of cell proliferation & 89 & $5.75 \mathrm{E}-08$ \\
\hline GO:0010941 & Regulation of cell death & 86 & $1.89 \mathrm{E}-06$ \\
\hline GO:0042981 & Regulation of apoptosis & 85 & 2.04E-06 \\
\hline GO:0007049 & Cell cycle & 78 & $3.50 \mathrm{E}-05$ \\
\hline GO:0001666 & Response to hypoxia & 22 & $8.73 \mathrm{E}-05$ \\
\hline GO:0015629 & Actin cytoskeleton & 48 & $1.01 \mathrm{E}-10$ \\
\hline GO:0044459 & Plasma membrane part & 207 & $2.19 \mathrm{E}-10$ \\
\hline GO:0044421 & Extracellular region part & 98 & \\
\hline GO:0003779 & Actin binding & 52 & $2.20 \mathrm{E}-10$ \\
\hline GO:0008092 & Cytoskeletal protein binding & 67 & $1.23 \mathrm{E}-09$ \\
\hline GO:0005516 & Calmodulin binding & 23 & $2.90 \mathrm{E}-05$ \\
\hline GO:0019865 & Immunoglobulin binding & 7 & $1.42 \mathrm{E}-04$ \\
\hline GO:0005524 & ATP binding & 105 & 0.043192 \\
\hline GO:0030695 & GTPase regulator activity & 35 & 0.028338 \\
\hline GO:0005088 & Ras guanyl-nucleotide exchange factor activity & 11 & 0.037291 \\
\hline
\end{tabular}

GO, gene ontology.

leukocyte transendothelial migration and cell adhesion molecule (Table III).

Verification of the differential genes by RT-qPCR. In order to verify the reliability of microarray results, the AD-associated genes, MYLK, MYH11, SOD3, FLNA, PKD-1 and TAGLN were screened according to their expression and GO classification using RT-qPCR; the results are shown in Table IV. Compared with the normal aortic tissue specimens, the mRNA expression in MYLK, MYH11, SOD3, PKD-1 and TAGLN genes in the AD group was significantly decreased $(\mathrm{P}<0.05)$, while that in FLNA was decreased, although the difference was not statistically significant.

\section{Discussion}

Investigations into the pathogenesis of $\mathrm{AD}$ have long been a major topic in academia. The pathogenesis of AD is considered to be a complex multifactorial process that involves several factors, including heredity (such as Marfan syndrome), hypertension, atherosclerosis, trauma, inflammation, oxidative stress and autoimmune diseases $(6,7)$. However, the mechanism by which these factors induce $\mathrm{AD}$ remains unclear. According to modern molecular biology, various external factors induce the occurrence and development of diseases by changing the gene expression profiles of tissues or cells. Thus, analyzing these changes enables us to understand the mechanism underlying the occurrence and development of the diseases at the molecular level. In the present study, the gene expression profiles of $\mathrm{AD}$ and normal aorta tissues were analyzed using human whole-genome microarray, and 1,661 genes with greater than 2 -fold differential expression were screened. These differential genes were involved in the cellular immune response, intracellular signaling cascades, and regulation of cell proliferation, cell death and apoptosis, as well as inflammatory reactions and oxidative stress in the cell cycle and other biological processes.

Inflammatory reactions occurring in the arterial wall are important in the incidence of cardiovascular diseases, including macrovascular atherosclerosis, myocardial infarction and congestive heart failure (8-10). He et al (11) employed anti-cluster of differentiation 3 (CD3) and anti-CD45 to evaluate the expression of T-lymphocytes in the aortic walls of patients with $\mathrm{AD}$, and used anti-CD68 to observe the expression of macrophages. Their results revealed that compared with normal controls, elevated expression levels of CD3, CD45 and CD68 were observed in the aortic walls of patients with $\mathrm{AD}$. In addition, macrophages are one of the earliest inflammatory cells invading the aorta, and they are predominantly involved in the reconstruction of the arterial wall by secreting metalloproteinases, collagenase, elastase and 
Table III. Kyoto Encyclopedia of Genes and Genomes biological pathways for differentially expressed genes.

\begin{tabular}{lrl}
\hline Pathway & Count & P-value \\
\hline Fc $\gamma$ R-mediated phagocytosis & 22 & $7.02 \mathrm{E}-06$ \\
Chemokine signaling pathway & 33 & $1.09 \mathrm{E}-05$ \\
Complement and coagulation cascades & 17 & $4.73 \mathrm{E}-05$ \\
Viral myocarditis & 17 & $6.86 \mathrm{E}-05$ \\
Leukocyte transendothelial migration & 22 & $2.08 \mathrm{E}-04$ \\
Type I diabetes mellitus & 12 & $2.33 \mathrm{E}-04$ \\
Allograft rejection & 11 & $2.66 \mathrm{E}-04$ \\
Cell adhesion molecules & 23 & $3.91 \mathrm{E}-04$ \\
Natural killer cell mediated cytotoxicity & 23 & $4.35 \mathrm{E}-04$ \\
Systemic lupus erythematosus & 19 & $4.48 \mathrm{E}-04$ \\
B cell receptor signaling pathway & 16 & $4.66 \mathrm{E}-04$ \\
Graft vs. host disease & 11 & $5.39 \mathrm{E}-04$ \\
Regulation of actin cytoskeleton & 30 & 0.001841243 \\
Intestinal immune network & 11 & 0.00348427 \\
for IgA production & & \\
Autoimmune thyroid disease & 11 & 0.004719241 \\
Hematopoietic cell lineage & 15 & 0.0054399 \\
Nucleotide-binding oligomerization & 12 & 0.006862366 \\
domain-like receptor signaling pathway & & \\
Cytokine-cytokine receptor interaction & 32 & 0.009531305 \\
Toll-like receptor signaling pathway & 16 & 0.009654688 \\
Dilated cardiomyopathy & 15 & 0.009906976 \\
p53 signaling pathway & 12 & 0.01367287 \\
Peroxisome proliferator-activated & 12 & 0.015182779 \\
receptors signaling pathway & & \\
Extracellular matrix-receptor & 13 & 0.025860442 \\
interaction & & \\
Hypertrophic cardiomyopathy & 13 & 0.02810487 \\
Focal adhesion & 24 & 0.033743536 \\
Fc $\gamma$ RI signaling pathway & 12 & 0.034919203 \\
Amyotrophic lateral sclerosis & 9 & 0.04755248 \\
\hline & & \\
& &
\end{tabular}

pro-inflammatory cytokines, which lead to aortic diseases. Yuan et al (12) observed the gene and protein levels of matrix metalloproteinase-2 (MMP-2) and MMP-9 in human AD specimens and identified macrophage-mediated chronic inflammation in the arterial wall. Tieu et al (13) developed an aortic reconstruction model by treating $\mathrm{ApoE}^{-/-}$mice with angiotensin II, and confirmed that interleukin (IL)-6 promoted the monocytes to differentiate into macrophages and induce cells to generate CD14 and CDllb, thereby increasing the expression levels of monocyte chemoattractant protein-1 and MMP-9. All these findings indicate that inflammatory factors are important in the incidence of AD. However, in the present study, 67 inflammation-associated genes exhibited significant differential expression in AD, of which IL-8, IL-6, IL-10, CD14, CD163 and secreted phosphoprotein 1, among others were significantly upregulated.

Previous studies have demonstrated that cardiovascular diseases, such as atherosclerosis, hypertension, atrial fibrillation, myocardial ischemia and cardiomyopathy are closely associated with reactive oxygen species. Liao et al (14) investigated the oxidative stress-associated indicators in the AD and normal aortic walls, and found that the malondialdehyde level in the $\mathrm{AD}$ group was significantly higher than in the normal aorta group, while the total SOD activity and genotype SOD activity were lower in the $\mathrm{AD}$ group than those in the normal aorta group. This indicated that the aortic wall of patients with $\mathrm{AD}$ was more severely attacked by free radicals and possessed a weaker ability to clear the free radicals when compared with the normal aortic wall, thereby suggesting oxidative stress injury in $\mathrm{AD}$ (14). The microarray results in the present study revealed that 22 oxidative stress-associated genes were significantly changed in the AD tissue samples. In addition, microarray analysis and RT-qPCR revealed that the expression of the SOD3 gene was significantly decreased in the $\mathrm{AD}$ group, which is consistent with previous results (14).

Similar results regarding genes that were significantly upregulated or downregulated in the AD tissue samples assessed in the present study have previously been reported, and studies have shown that certain genes were involved in various biological processes. For example, polycystic kidney disease gene-1-encoded protein product, namely polycystin-1 (PC-1), participates in cell adhesion and cell signal transduction. Hassane et al (15) conducted experiments using a PKD-1 knockout mouse model, and found that the downregulation of $P K D-1$ expression caused the mouse aorta to generate potential space and intramural hematoma, and led to a series of structural changes that are similar to those occurring prior to $\mathrm{AD}$. The microarray analysis and RT-qPCR validation performed in the present study also revealed significant downregulation of $P K D-1$ expression in patients with AD.

The medial layer of the aorta is predominantly composed of vascular smooth muscle cells and $45-55$ layers of elastin. Vascular smooth muscle cells are key in the maintenance of vascular homeostasis, regulation of blood pressure, and damage repair processes, and maintain the arterial tension through their own contractile function, as well as by synthesizing and secreting extracellular matrix (16). Thus, smooth muscle cell proliferation and phenotype modulation abnormalities are likely to alter the activities of extracellular matrix and proteolytic enzymes, and promote the characteristic structural diseases in the development of AD. Meanwhile, MYLK encoding the myosin light-chain kinase (MLCK) protein and the MYH11 gene are the genes encoding special myosins for smooth muscle cells, which are the important components of contractile units of smooth muscle cells $(17,18)$.

Previous study reported that certain human chronic and acute diseases are associated with increased MLCK expression levels or activity (19-21); MLCK was found to affect the damage caused by the inflammatory reaction by regulating the myosin light chain. MLCK regulates the contraction of smooth muscle cells (22) and is the key signaling pathway involved in the regulation of smooth muscle cell contraction. Furthermore, as a multifunctional regulator, MLCK has non-kinase actin and myosin-binding activity, and it is important in smooth muscle cell proliferation, migration and reconstruction of the cytoskeleton (23). Additionally, an MYH11 gene mutation was reported to alter the contraction of smooth muscle cells (24). In the present study, microarray analysis and RT-qPCR verified that the expression levels of MYLK and MYH11 were 


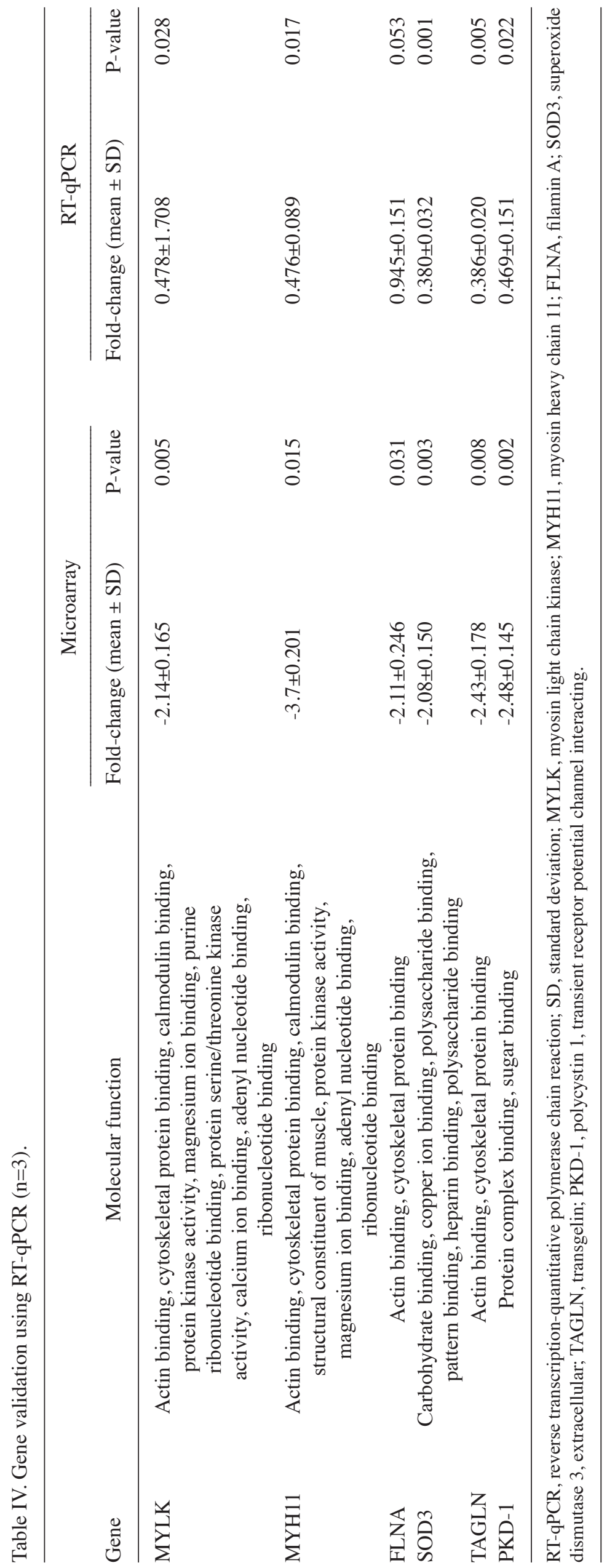


significantly downregulated in the AD tissue samples, and that they may be involved in the occurrence and development of $\mathrm{AD}$ by regulating the contraction of smooth muscle cells.

In addition, the current study predicted that certain genes, such as TAGLN and FLNA, might be associated with $\mathrm{AD}$, although, to the best of our knowledge, their role in the development of AD has not been thoroughly investigated. Microarray analysis revealed that the expression levels of these two genes were significantly downregulated; however, RT-qPCR confirmed that FLNA was downregulated in the AD group without statistical significance, compared with the normal group.

In the present study, high-density whole genome expression microarray was used to identify differentially expressed genes between $\mathrm{AD}$ and healthy aorta tissue samples, as well as to analyze the function, location, and other data regarding the differential genes. The results revealed that 997 genes were upregulated and 664 genes were significantly downregulated in the pathogenesis of AD. In addition, six differential genes were selected for verification by RT-qPCR, which indicated that the expression levels of these six genes were downregulated in the $\mathrm{AD}$ group compared with the healthy aorta group, five of which were significantly downregulated $(\mathrm{P}<0.05)$ and that FLNA was downregulated, although it was not statistically significant $(\mathrm{P}>0.05)$. In conclusion, these findings demonstrate that the genes screened by microarray were closely associated with the pathogenesis of $\mathrm{AD}$, providing a basis for further investigation of the molecular mechanism underlying the pathogenesis of AD.

\section{Acknowledgements}

The present study was supported by the National Natural Science Foundation of China (grant no. 81270385).

\section{References}

1. Mészáros I, Mórocz J, Szlávi J, Schmidt J, Tornóci L, Nagy L and Szép L: Epidemiology and clinicopathology of aortic dissection. Chest 117: 1271-1278, 2000.

2. Wang S, Wang J, Lin P, Li Z, Yao C, Chang G, Li X and Wang S: Short-term curative effect of endovascular stent-graft treatment for aortic diseases in China: A systematic review. PLoS One 8: e71012, 2013

3. Gandet T, Canaud L, Ozdemir BA, Ziza V, Demaria R, Albat B and Alric P: Factors favoring retrograde aortic dissection after endovascular aortic arch repair. J Thorac Cardiovasc Surg 150: 136-142, 2015.

4. Wang F, Li B, Lan L and Li L: C596G mutation in FBN1 causes Marfan syndrome with exotropia in a Chinese family. Mol Vis 21: 194-200, 2015.

5. Reinstein E, DeLozier CD, Simon Z, Bannykh S, Rimoin DL and Curry CJ: Ehlers-Danlos syndrome type VIII is clinically heterogeneous disorder associated primarily with periodontal disease, and variable connective tissue features. Eur J Hum Genet 21: 233-236, 2013.

6. Scali ST, Waterman A, Feezor RJ, Martin TD, Hess PJ Jr, Huber TS and Beck AW: Treatment of acute visceral aortic pathology with fenestrated/branched endovascular repair in high-surgical-risk patients. J Vasc Surg 58: 56-65.e1, 2013.
7. Faure EM, Canaud L, Agostini C, Shaub R, Böge G, Marty-ané C and Alric P: Reintervention after thoracic endovascular aortic repair of complicated aortic dissection. J Vasc Surg 59: 327-333, 2014.

8. Wales KM, Kavazos K, Nataatmadja M, Brooks PR, Williams C and Russell FD: N-3 PUFAs protect against aortic inflammation and oxidative stress in angiotensin II-infused apolipoprotein E-/mice. PLoS One 9: e112816, 2014.

9. Brasier AR: The nuclear factor-kappaB-interleukin-6 signalling pathway mediating vascular inflammation. Cardiovasc Res 86: 211-218, 2010.

10. Maiellaro $\mathrm{K}$ and Taylor WR: The role of the adventitia in vascular inflammation. Cardiovasc Res 75: 640-648, 2007.

11. He R, Guo DC, Estrera AL, Safi HJ, Huynh TT, Yin Z, Cao SN, Lin J, Kurian T, Buja LM, et al: Characterization of the inflammatory and apoptotic cells in the aortas of patients with ascending thoracic aortic aneurysms and dissections. J Thorac Cardiovasc Surg 131: 671-678, 2006.

12. Yuan Y, Wang C, Xu J, Tao J, Xu Z and Huang S: BRG1 overexpression in smooth muscle cells promotes the development of thoracic aortic dissection. BMC Cardiovasc Disord 14: 144, 2014.

13. Tieu BC, Lee C, Sun H, Lejeune W, Recinos A III, Ju X, Spratt H, Guo DC, Milewicz D, Tilton RG, et al: An adventitial IL-6/MCP1 amplification loop accelerates macrophage-mediated vascular inflammation leading to aortic dissection in mice. J Clin Invest 119: 3637-3651, 2009.

14. Liao M, Liu Z, Bao J, Zhao Z, Hu J, Feng X, Feng R, Lu Q, Mei Z, Liu Y, et al: A proteomic study of the aortic media in human thoracic aortic dissection: implication for oxidative stress. J Thorac Cardiovasc Surg 136: 65-72, 72.e1-3, 2008.

15. Hassane S, Claij N, Lantinga-van Leeuwen IS, Van Munsteren JC, Van Lent N, Hanemaaijer R, Breuning MH, Peters DJ and DeRuiter MC: Pathogenic sequence for dissecting aneurysm formation in a hypomorphic polycystic kidney disease 1 mouse model. Arterioscler Thromb Vasc Biol 27: 2177-2183, 2007.

16. Ito S, Ozawa K, Zhao J, Kyotani Y, Nagayama K and Yoshizumi M: Olmesartan inhibits cultured rat aortic smooth muscle cell death induced by cyclic mechanical stretch through the inhibition of the c-Jun N-terminal kinase and p38 signaling pathways. J Pharmacol Sci 127: 69-74, 2015.

17. Wang L, Guo DC, Cao J, Gong L, Kamm KE, Regalado E, Li L, Shete S, He WQ, Zhu MS, et al: Mutations in myosin light chain kinase cause familial aortic dissections. Am J Hum Genet 87: 701-707, 2010.

18. Bellini C, Wang S, Milewicz DM and Humphrey JD: Myh11(R247C/R247C) mutations increase thoracic aorta vulnerability to intramural damage despite a general biomechanical adaptivity. J Biomech 48: 113-121, 2015.

19. Chen D, Lin Y and Xiong Y: Epithelial MLCK and smooth muscle MLCK may play different roles in the development of inflammatory bowel disease. Dig Dis Sci 59: 1068-1069, 2014.

20. Zhang W, Cheng Z, Qu X, Dai H, Ke X and Chen Z: Overexpression of myosin is associated with the development of uterine myoma. J Obstet Gynaecol Res 40: 2051-2057, 2014.

21. Zou DB, Wei X, Hu RL, Yang XP, Zuo L, Zhang SM, Zhu HQ, Zhou Q, Gui SY and Wang Y: Melatonin inhibits the Migration of Colon Cancer RKO cells by Down-regulating Myosin Light Chain Kinase Expression through Cross-talk with p38 MAPK. Asian Pac J Cancer Prev 16: 5835-5842, 2015.

22. Milewicz DM, Guo DC, Tran-Fadulu V, Lafont AL, Papke CL, Inamoto S, Kwartler CS and Pannu H: Genetic basis of thoracic aortic aneurysms and dissections: Focus on smooth muscle cell contractile dysfunction. Annu Rev Genomics Hum Genet 9: 283-302, 2008

23. Betapudi V: Life without double-headed non-muscle myosin II motor proteins. Front Chem 2: 45, 2014.

24. Renard M, Callewaert B, Baetens M, Campens L, MacDermot K, Fryns JP, Bonduelle M, Dietz HC, Gaspar IM, Cavaco D, et al: Novel MYH11 and ACTA2 mutations reveal a role for enhanced TGF $\beta$ signaling in FTAAD. Int J Cardiol 165: 314-321, 2013. 